\title{
Research on Taguchi TOPSIS Method in Logistics Service Quality
}

\author{
TANG Miao ${ }^{1}$,Wang Tie-dan ${ }^{2}$, Peng Ding-hong ${ }^{3 *}$ \\ Quality Development Institute, Kunming University of Science and Technology \\ Kunming 650093, China
}

\begin{abstract}
With the rapid development of e-commerce, the logistics industry has also received much attention. Therefore, how to choose the best service quality logistics enterprise from a large number of logistics enterprises has become the primary problem faced by enterprise users. In order to select the highest service quality logistics enterprise and improve the stability of service quality, this study applies the Taguchi method to the research of logistics service quality. The TOPSIS method is used to analyze the data, and the Taguchi and TOPSIS methods are integrated to make the best choice. Finally, the rationality of the conclusion of this paper is verified by a related example.
\end{abstract}

Keywords-Logistics service quality; Taguchi method; TOPSIS; Multiple attribute decision

\section{INTRODUCTION}

In recent years, outsourcing services such as network services, financial services and logistics services have seen considerable profits and are increasingly popular. Many service providers are paying more and more attention to the quality of service in order to meet customer needs. Especially in the logistics service, not only tailor-made exclusive services, but also the pursuit of personalized services, professional business, etc., so that they stand out in the competitive position, so the evaluation of logistics service quality is particularly important. At present, logistics service quality research has attracted the attention of many scholars as a hot issue in service quality research. Limbourg [1] and others used SERVQUAL to evaluate the quality of logistics services by analyzing specific case themes. Rahmat [2] and others analyzed the impact of suppliers' satisfaction with the quality of logistics services in terms of operations, relationships and national culture. Using the fair entropy function, Liu et al[3]. proposed a logistics enterprise based on the optimal quantity decision method of logistics service quality defect guarantee and return fairness to select the best service quality. Although the above methods have enriched the research on the evaluation of logistics service quality, it still needs to be broken. Traditionally, the evaluation of logistics service quality relies on a large amount of data to carry out related calculations or research on corporate culture, and does not correctly reflect the real influencing factors of logistics service quality. And with the diversification of people's thinking, in order to ensure the authenticity of the evaluation results, some scholars have proposed hesitant fuzzy sets to fully describe the decision information.Therefore, this paper uses the Taguchi method, an advanced tool in the field of quality engineering, combined with the TOPSIS classical decision-making method, through the standardization and weighting of the decision matrix determines the positive and negative ideal schemes and calculates the signal-to-noise ratio of the positive and negative ideal schemes. Sort the decision schemes to ensure the validity of the decision information.It provides a reference for logistics companies that choose the best service quality.

\section{RESEARCH METHODOLOGY}

\section{A. Taguchi method}

In the early 1970s, Genichi Taguchi founded the Taguchi method. The core of the Taguchi method was based on orthogonal design, and the introduction of signal noise ratio reduced the influence of error on the test results[4]. The Taguchi method is a low-cost, high-efficiency optimization method for quality engineering. It emphasizes that the improvement of product quality is not through inspection, but through design.

1) Orthogonal table. Orthogonal table is a standardized table that obtains more comprehensive information with less trials, using $L_{q}\left(t^{m}\right)$, where $L$ orthogonal table symbols, $q$ is the number of trials, $t$ is the number of factors, and $m$ is the participation of the test. factor.

In the Taguchi method, firstly, according to the number of initial experimental participation factors, the corresponding twolevel orthogonal table is selected, and the feature vector is placed in the 2-level orthogonal table. The 2-level orthogonal table represents two experimental factors. Characterization. Where " " represents the selection of the feature vector and " " represents that the feature vector is not selected. Commonly used 2 horizontal orthogonal tables have: $L_{4}\left(2^{3}\right) 、 L_{8}\left(2^{7}\right)$. $L_{16}\left(2^{15}\right), L_{32}\left(2^{31}\right)$, etc. 
For example: 2 horizontal orthogonal table, if optimized by orthogonal table, only 8 trials can get more comprehensive information, as shown in Table 1.

TABLE I HORIZONTAL ORTHOGONAL TABLE

\begin{tabular}{cccccccc}
\hline test & $c_{1}$ & $c_{2}$ & $c_{3}$ & $c_{4}$ & $c_{5}$ & $c_{6}$ & $c_{7}$ \\
\hline$A$ & 1 & 1 & 1 & 1 & 1 & 1 & 1 \\
$B$ & 1 & 1 & 1 & 2 & 2 & 2 & 2 \\
$C$ & 1 & 2 & 2 & 1 & 1 & 2 & 2 \\
$D$ & 1 & 2 & 2 & 2 & 2 & 1 & 1 \\
$E$ & 2 & 1 & 2 & 1 & 1 & 1 & 2 \\
$F$ & 2 & 1 & 2 & 2 & 2 & 2 & 1 \\
$G$ & 2 & 2 & 1 & 1 & 2 & 2 & 1 \\
$H$ & 2 & 2 & 1 & 2 & 1 & 1 & 2 \\
\hline
\end{tabular}

2) Signal-to-Noise Ratio. The signal-to-noise ratio is used to measure the output response of each test, denoted by $\eta$. As a functional evaluation index, the signal-to-noise ratio has three functions: the selection of characteristic variables under specific conditions, the function of the measurement system, and the number of measured characteristic variables. It is mainly divided into three categories: Nominal is best (NB), Higher is better (HB) and Lower is better (LB). In this paper, we choose the small signal-to-noise ratio. The smaller the $\eta$ value, the more stable the quality.

$$
\eta_{S N R L \bar{B}}-10 \log \left[\frac{1}{q} \sum_{k=1}^{q} d k(]\right.
$$

$d(k)$ is the distance from the $k$ th sample to the whole 。

\section{B. TOPSIS}

TOPSIS (Technique for Order Preference by Similarity to an Ideal Solution) has received much interest from researchers and practitioners as a popular Multi-Criteria Decision Making (MCDM) method $^{[5]}$. At present, the TOPSIS method is widely used in multiattribute decision making such as ERP selection and real estate evaluation. Therefore, it is not only necessary to introduce the TOPSIS method into the service quality evaluation of logistics enterprises, but also to scientifically and effectively select and evaluate multi-attribute decision-making schemes.

At present, the TOPSIS method is widely used in multi-attribute decision-making such as ERP selection, real estate evaluation and investment evaluation of other large-scale projects. The evaluation of service quality of logistics enterprises has commonality with the above projects. Therefore, it is not only necessary to introduce the TOPSIS method into the service quality evaluation of logistics enterprises, but also to scientifically and effectively select and evaluate multi-attribute decision-making schemes.

\section{Hesitation Fuzzy Set}

Torra first introduced the Hesitation Fuzzy Set (HFS), which allows the membership of an element to be represented as several possible values between 0 and 1 [8].

Definition 1 [8] Assumes that $x$ is a given set, $x$ and the HFS on $x$ returns a subset of $[0,1]$ based on the function applied to $\mathrm{B}$, which can be expressed as the following mathematical notation:

$$
E=\left\{\left\langle x, h_{E}(x)\right\rangle \mid x \in X\right\}
$$

Where $h_{E}(x)$ is a set of values in $[0,1]$, indicating the possible membership of element $x \in X$ on set $E$, and $h_{E}(x)$ is a hesitant fuzzy element (HFE).

\section{HESITANT FUZZY TAGUCHI TOPSIS METHOD}

With the hesitation of human thinking and increasingly complex decision-making problems, it is difficult for policy makers to use accurate numbers to represent decision information. However, the classical TOPSIS method is difficult to realize the increasingly complex decision-making problem. Therefore, this paper proposes a fuzzy multi-attribute decision-making method, which firstly standardizes the weighted decision matrix, weights the process, determines the positive and negative ideal solution, and calculates the positive and negative ideal solution. The noise ratio is finally sorted according to the closeness. The hesitant fuzzy Taguchi TOPSIS sorting method is as follows:

Step 1: Construction decision matrix.

$$
H=\left(\begin{array}{cccc}
h_{11} & h_{12} & \ldots & h_{1 n} \\
h_{21} & h_{22} & \ldots & h_{2 n} \\
\ldots & \ldots & \ldots & \ldots \\
h_{m 1} & h_{m 2} & \ldots & h_{m n}
\end{array}\right)_{m \times n}
$$

Step 2: Normalized decision matrix. $H^{\prime}=\left(h_{i j}^{\prime}\right)_{n \times m}$

Benefit type: $h_{i j}=h_{i j} \quad$ Cost type: $h_{i j}=h_{i j}^{c}$

Step 3: Determine positive and negative ideal solutions.

$$
\begin{aligned}
& P_{+}=\max _{1 \leq \tilde{h}_{i j} \leq n}\left\{h_{i 1}^{+}, h_{i 2}^{+}, \ldots, h_{i n}^{+}\right\} \\
& P_{-}=\min _{1 \leq \tilde{h}_{i j} \leq n}\left\{h_{i 1}, h_{i 2}, \ldots, h_{i n}\right\}
\end{aligned}
$$

Step 4: Calculation scheme and positive and negative ideal solution distance. This paper uses the Hamming distance of $\mathrm{XU}^{[6]}$ to calculate the distance between two hesitant fuzzy elements.

$$
d\left(h_{1}, h_{2}\right)=w_{i} \frac{1}{l} \sum_{\lambda=1}^{l}\left|\gamma_{1}^{\lambda}-\gamma_{2}^{\lambda}\right|
$$

Step 5: Calculating the signal to noise ratio.

$$
\eta_{S N R . L B}=-10 \log _{10}\left[\frac{1}{q} \sum_{k=1}^{q} d(k)\right]
$$

Step 6: Calculate closeness.

$$
\beta_{i}=\frac{\eta_{i}^{-}}{\eta_{i}^{-}+\eta_{i}^{+}}
$$

Step 7: Final sorting of the scheme.

\section{CASE AnAlysis}

Quality of service is critical to the growing logistics industry. The existing four logistics enterprises are respectively denoted as $\left\{P_{1}, P_{2}, P_{3}, P_{4}\right\}$. In order to select the best service quality, the logistics enterprises are evaluated from four evaluation indicators $\left\{c_{1}, c_{2}, c_{3}, c_{4}\right\}:$ individualization, information technology, 
innovation development and specialization, as shown below in figure I. The corresponding weight is $\quad w=(0.35,0.28,0.1,0.27)$. The benefit indicator, so the normalized decision matrix is consistent with the initial decision matrix.

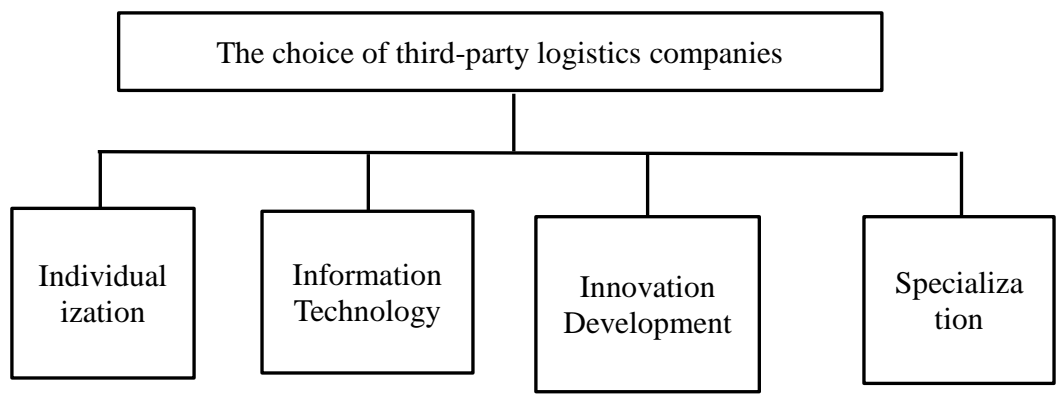

Fig. 1 Evaluation indicators selected by third-party logistics companies

TABLE II HESITANT FUZZY DECISION MATRIX

\begin{tabular}{lllll}
\hline & $c_{1}$ & $c_{2}$ & $c_{3}$ & $c_{4}$ \\
\hline$P_{1}$ & $\{0.3,0.5\}$ & $\{0.5\}$ & $\{0.2,0.1\}$ & $\{0.4,0.6\}$ \\
$P_{2}$ & $\{0.4,0.7\}$ & $\{0.3,0.4\}$ & $\{0.2,0.5\}$ & $\{0.7,0.9\}$ \\
$P_{3}$ & $\{0,2,0,3\}$ & $\{0.4\}$ & $\{0.5,0.6\}$ & $\{0.3,0.4\}$ \\
$P_{4}$ & $\{0.5,0.6\}$ & $\{0.5,0.8\}$ & $\{0.3,0.6\}$ & $\{0.6,0.7\}$ \\
\hline
\end{tabular}

The positive and negative ideal solutions are determined according to formula (5)(6):

$$
\begin{aligned}
& P^{+}=\{\{0.5,0.7\},\{0.5,0.8\},\{0.5,0.6\},\{0.7,0.9\}\} \\
& P^{-}=\{\{0.2,0.3\},\{0.3,0.4\},\{0.2,0.1\},\{0.3,0.4\}\}
\end{aligned}
$$

The Hamming distances from the solution to the positive and negative ideal solutions are:

TABLE III DISTANCE FROM EACH SCHEME TO THE POSITIVE IDEAL SOLUTION

\begin{tabular}{ccccc}
\hline & $d_{1}$ & $d_{2}$ & $d_{3}$ & $d_{4}$ \\
\hline$P_{1}$ & 0.0700 & 0.0350 & 0.0400 & 0.0810 \\
$P_{2}$ & 0.0175 & 0.0840 & 0.0200 & 0 \\
$P_{3}$ & 0.1225 & 0.0420 & 0 & 0.1215 \\
$P_{4}$ & 0.0175 & 0 & 0.0100 & 0.0405 \\
\hline
\end{tabular}

TABLE IV DISTANCE FROM EACH SCHEME TO THE NEGATIVE IDEAL SOLUTION

\begin{tabular}{ccccc}
\hline & $d_{1}$ & $d_{2}$ & $d_{3}$ & $d_{4}$ \\
\hline$P_{1}$ & 0.0525 & 0.0490 & 0 & 0.0405 \\
$P_{2}$ & 0.1225 & 0 & 0.0200 & 0.1215 \\
$P_{3}$ & 0 & 0.0350 & 0.0400 & 0 \\
$P_{4}$ & 0.1050 & 0.0840 & 0.0300 & 0.0810 \\
\hline
\end{tabular}

TABLE V POSITIVE AND NEGATIVE IDEAL SOLUTION SIGNAL TO NOISE RATIO

\begin{tabular}{cccccccc}
\hline$\eta_{1}^{+}$ & $\eta_{2}^{+}$ & $\eta_{3}^{+}$ & $\eta_{4}^{+}$ & $\eta_{1}^{-}$ & $\eta_{2}^{-}$ & $\eta_{3}^{-}$ & $\eta_{4}^{-}$ \\
\hline 12.48 & 15.17 & 11.46 & 17.70 & 14.50 & 11.81 & 17.26 & 11.25 \\
\hline
\end{tabular}

According to the closeness calculation, the final result is as follows: $P_{1}=0.537, P_{2}=0.438, P_{3}=0.601, P_{4}=0.389$, $P_{3}>P_{1}>P_{2}>P_{4}$. The research method of this paper thinks that the service quality of the third logistics enterprise is better than the other three, and it is more worthy of choice. 


\section{CONCLUSION}

In this paper, we use the two key tools of Taguchi method to study the quality of logistics service from the perspective of abstract decision-making indicators.This paper proposes a hesitant fuzzy Taguchi TOPSIS multi-attribute decision making method. This method avoids the loss of effective decision information and makes the decision result more in line with people's true intention. When applying Taguchi TOPSIS method to solve the logistics service quality, the quality fluctuation is fully considered. Sexuality solves the problem of unreasonable quality of traditional evaluation services. It is used as a reference for multi-attribute decision making for Taguchi method.

\section{REFERENCES}

[1] Limbourg S, Giang H T Q, Cools M. Logistics Service Quality: The Case of Da Nang City [J]. Procedia Engineering, 2016, 142:124-130.

[2] Rahmat A K, Faisol N. Manufacturers Satisfaction on Logistics Service Quality: Operational, Relational and National Culture is [J]. Procedia Social and Behavioral Sciences, 2016, 224:339-346.

[3] Liu W, Shen X, Xie D. Decision Method for the Optimal Number of Logistics Service Providers with Service Quality Guarantee and Revenue Fairness [J]. Applied Mathematical Modelling, 2017, 48.

[4] Mahalanobis P C.On the generalized distance in statistics[C]//Proceedings of the National Institute of Sciences of India,1936(2): 49-55.

[5] Behzadian M, Otaghsara S K, Yazdani M, et al. A state-of the-art survey of TOPSIS applications[J]. Expert Systems with Applications, 2012, 39(17): 13051-13069.

[6] Xu Z S, Xia M M. Distance and similarity measures for hesitant fuzzy sets [J]. Information Sciences, 2011,181(11): 2128-2138. 